\title{
Violencia hacia las otras formas de ser mujer y de ser nombre*
}

\author{
Andrea Marcela Mahecha Montañez \\ Universidad Distrital Francisco José de Caldas (DIE), Bogotá, Colombia \\ andreamahecha@uan.edu.co \\ Jonathan David Varela \\ Universidad Antonio Nariño, Bogotá, Colombia \\ jvarela43@uan.edu.co \\ https://orcid.org/0000-0003-4486-4989
}

\section{RESUMEN}

El presente escrito es el resultado del normatividad. A partir de una metodología ejercicio investigativo que indagó por las nuevas feminidades y masculinidades presentes en los grados décimos del Colegio Distrital Paulo Freire de la localidad de Usme, Bogotá. Para ello, se analizaron las violencias de las que son víctimas los y las estudiantes que construyen su identidad de género por fuera de los esquemas tradicionales establecidos desde la heterocualitativa, se hizo uso de la encuesta y la entrevista para identificar estereotipos de género junto con las formas de violencia que circulan en el espacio escolar. Tales datos fueron analizados con ayuda de la herramienta informática Atlas.ti.

Palabras clave: feminidades; masculinidades; heteronormatividad; género; escuela.

Cómo citar: Maecha, A. y Varela, J. (2021). Violencia hacia las otras formas de ser mujer y de ser hombre. Ciencias Sociales y Educación, 10(19), 197-216, https://doi.org/10.22395/csye.v9n18a9

Recibido: 13 de marzo de 2020.

Aprobado: 17 de julio de 2020. 


\section{Violence Towards the Other Ways of Being woman and Man}

\section{ABSTRACT}

This piece of writing is the result of a research exercise that inquired on new femininities and masculinities within the 10th grades of the Paulo Freire District School in the Usme locality, Bogotá. For that, the research analyzed the violence that these students are victims of for constructing their gender identities outside of the traditional structures established by heteronor- mativity. With a qualitative methodology, the study performed polls and interviews for identifying gender stereotypes with the forms of violence circulating in the school space. The data was analyzed with Atlas. ti software tool.

Keywords: femininities; masculinities; heteronormativity; gender; school.

\section{Violência contra outras formas de ser mulher e de ser homem}

\section{RESUMO}

Este trabalho é o resultado do exercímetodologia qualitativa, fez-se uso da cio investigativo que questionou as novas feminilidades e masculinidades presentes no ensino médio do Colégio Distrital Paulo Freire, na cidade de Usme, Bogotá. As diversas formas de violência que os alunos sofrem e que constroem sua identidade de gênero, fora dos esquemas tradicionais estabelecidos a partir da heteronormatividade, foram analisadas. A partir de uma pesquisa e da entrevista para identificar os estereótipos de gênero e as formas de violência que circulam no espaço escolar. Esses dados foram analisados com o auxílio da ferramenta computacional Atlas.ti.

Palavras-chave: escola; feminilidades; gênero; heteronormatividade; masculinidades. 


\section{Introducción}

La teoría de la reproducción, originariamente del pensamiento sociológico, tuvo un impacto significativo en la forma de pensar la educación a finales de los sesenta y principios de los setenta, por su carácter crítico frente a la escuela como una institución cuya función es la reproducción del sistema capitalista. Sin embargo, para las décadas de los setenta y los ochenta, se proyecta una crítica sobre ella que fundamentará la siguiente teoría. La teoría de la resistencia aparece, así, como oposición a la teoría de la reproducción por su carácter determinista y por su carencia en proposición a algún cambio. Por ello, centrará su atención en las formas de resistencia en el escenario escolar para la promoción de un proyecto de transformación social (Hirsch y Rio, 2015).

Entre sus figuras académicas representativas, se destacan el pedagogo Henry Giroux (1983), quien, reconociendo los aportes brindados por la teoría de la reproducción para el ámbito educativo, sostiene que dicho modelo teórico ha enfatizado tanto el aspecto de dominación de la escuela que ha ignorado las contradicciones que allí se gestan. Siguiendo planteamientos de la teoría de la resistencia, explica que los mecanismos que funcionan para la reproducción social nunca llegan a cometer su objetivo completamente, puesto que existen formas que se le oponen. Por lo tanto, se entiende la dominación como una relación dinámica que no llega a completarse en su totalidad. Por lo anterior, puede identificarse la escuela como un espacio dialéctico, donde se enfrentan fuerzas ideológicas que promueven determinados proyectos de sociedad. Así, aunque su rol parezca inclinarse mayoritariamente a su aspecto reproductivo, no debe descartarse la capacidad de agenciamiento que permite llevar a cabo cambios necesarios en la estructura educativa.

Teniendo en cuenta lo anterior, el siguiente trabajo propone exponer las formas violentas en las que se reproduce el sistema de dominación heterosexual en la escuela como una arista del sistema capitalista. Se parte de la idea de que cualquier intento por trasformar la institución educativa para mitigar los efectos violentos provenientes de los sistemas de dominación, necesita identificar la manera en la que estos se materializan. Así pues, se tratará de identificar, evidenciar y exponer una problemática que a simple vista pasa desapercibida, pero necesita ser atendida. Con ello, se espera abrir el campo reflexivo sobre las posibles soluciones pedagógicas que puedan desarrollarse para mejorar las relaciones entre las subjetividades que circulan en el espacio escolar.

Entre los personajes representantes de la teoría de la reproducción, se encuentra Louis Althusser (2003), quien, siguiendo la afirmación planteada por Marx de que la finalidad de la producción es la reproducción de las condiciones que permiten su mantenimiento, esto es, de las fuerzas productivas 
y de las relaciones de producción, se interesará por identificar la forma en la que el sistema capitalista se reproduce. Para ello, presta atención al Estado. Aunque lo concibe como un aparatado represivo que usa la clase burguesa para el dominio de la clase proletaria, supera dicha visión clásica marxista del Estado represivo para hablar de otros mecanismos que permiten su funcionamiento, los cuales define como aparatos ideológicos del Estado. Representados mediante las instituciones sociales, los aparatos ideológicos del Estado ponen en marcha la reproducción del sistema capitalista, no mediante el uso de la fuerza, sino por la ideología.

La ideología, tal como lo plantea Althusser (2003), no es la representación de las relaciones de producción, sino la relación imaginaria de los sujetos con las relaciones de producción. De esta manera, lo que se representa no es la realidad en sí misma, sino la deformación de ella, en la que se esconden determinados intereses de clase. Cabe mencionar que la ideología no queda circunscrita al mero conglomerado de ideas, pues tiene la capacidad de materializarse mediante las prácticas realizadas por los sujetos, las cuales son orientadas por las ideas que configuran el mismo plano ideológico.

Entre los aparatos ideológicos del Estado moderno se privilegia la escuela porque es un lugar donde se aprenden valores como la sumisión u dominación, así como las habilidades que permitirán el desenvolvimiento en el futuro trabajo, lo que implica la posición en una determinada clase social a la vez que la incorporación en las relaciones de producción. Mediante las instituciones sociales, primordialmente la escuela, se logra que los sujetos acepten el statu quo y, por ende, su propio sometimiento. De esta forma, Althusser (2003) devela la supuesta neutralidad de la escuela y descubre que está permeada de ideología que permite la reproducción de la explotación capitalista.

Ahora bien, si Althusser superó la visión clásica del marxismo sobre el Estado, también se puede hacer lo mismo con su concepción marxista sobre la escuela. En este sentido, la escuela no solo representa un espacio donde se reproduce una forma de dominación, a saber, la capitalista, sino también otras modalidades de poder más allá de la clase, pero que mantienen articulación con ella para su funcionamiento. Hablamos, pues, de las relaciones de dominio que se efectúan en torno a la raza, el sexo, la sexualidad, la nacionalidad, etc. De esta forma, se puede avanzar sobre la lectura de la escuela como un mecanismo que perpetua el capitalismo para concebirla también como una institución social. La interacción que se lleva a cabo por parte de los sujetos que frecuentan esta institución desarrolla, en ocasiones, relaciones de desigualdad que reflejan la manifestación de los regímenes opresivos que estructuran la sociedad. 
En el presente estudio nos enfocaremos en identificar las formas de violencia que produce el sistema heterosexual y que está latentes en un colegio público de Bogotá. Uno de los estudios predecesores con relación a la temática en mención es el que desarrolló el antropólogo Erik Cantor (2009), quien indagó a cerca de las expresiones de homofobia "a nivel emocional, comportamental y cognitivo predominantes entre los y las estudiantes de secundaria" (2009, p. 101), y las consecuencias que dicho fenómeno acarrea para la población estudiantil homosexual. Con base en los datos analizados, Cantor (2009) identificó, con relación a la homofobia cognitiva, que las representaciones mentales estudiantiles acerca de las personas homosexuales giran en torno a: lo antinatural, por no ser una sexualidad originaria de la naturaleza divina, esto es, de la creación del hombre y la mujer (sobrentiéndase heterosexuales) por parte de dios; lo anormal, tanto en el campo biológico como social. La anormalidad biológica de la homosexualidad, por un lado, radicaría en problemas genéticos u hormonales. La anormalidad social, por otro lado, radicaría en la ausencia de una sexualidad normal por situaciones difíciles que han logrado marcar a los sujetos de manera negativa: violación, maltrato infantil o fracaso amoroso. Y lo peligroso de que se proyecte la promiscuidad como una característica de la homosexualidad, es que parte del estudiantado cree que tal hecho conllevaría a cometer actos de violencia sexual.

En cuanto a la homofobia emocional, se identificaron las emociones: asco, debido a que la actividad sexual por parte de homosexuales supera los límites del sexo definidos por la heterosexualidad, esto es, las supuestas únicas zonas erógenas del cuerpo que paralelamente funcionan como órganos reproductivos; miedo, por la posible inclinación que pueden tener los y las homosexuales por querer entablar una relación amorosa o sexual con sus pares heterosexuales; por último, se encuentran las emociones de la rabia y odio. Pese a que no se presentan las causas de las última, se presenta su materialización mediante las prácticas violenta. Este hecho nos convoca a hablar de la homofobia comportamental. Esta se manifiesta mediante la burla, el rechazo y la agresión física, ejercidas mayormente por los hombres que por las mujeres. La razón de tales comportamientos, como lo señala Cantor (2009), se debe al desconocimiento de la diversidad sexual y/o falta de un vínculo o relación con una persona homosexual.

Entre las consecuencias que puede ocasionar el anterior contexto escolar en la población estudiantil homosexual, se encuentra la limitación por entablar una relación estable, lo que en ocasiones conduce a optar por encuentros sexuales esporádicos que aumentan las probabilidades de contraer una enfermedad sexual; el aislamiento social con la finalidad de evitar conflictos o incluso la deserción escolar si estos se hacen cada vez más latentes; la reproducción de la violencia, ya que para algunas víctimas la hostilidad se convierte en un 
mecanismo de defensa; el consumo de drogas como una forma de refugio y, en el peor de los casos, ideas y actitudes suicidas.

Las escuelas, en vez de afrontar dichas cuestiones, muchas veces las ignoran. Así, contribuyen de manera implícita a la reproducción de violencias que ayudan a mantener vigente un sistema específico de dominación: la heteronormatividad. De allí la importancia que adquiere observar a la institución educativa con enfoques como el género y la sexualidad, pues actúan como lentes analíticos que proporcionan la facultad de identificar problemáticas que no pueden ser reconocidas a simple vista, pero que necesitan ser atendidas con urgencia.

Teniendo en cuenta las anteriores consideraciones, el presente trabajo se interesó por indagar otras feminidades y masculinidades que coinciden en el escenario escolar, es decir, aquellas formas de ser mujer y de ser hombre que no reproducen los estereotipos hegemónicos y clásicos de la feminidad y la masculinidad. Este análisis se hizo en dos grados de décimo del colegio Paulo Freire, ubicad en la ciudad de Bogotá con el fin de identificar cuáles son las manifestaciones de violencia que se materializan en tales estudiantes para desafiar la normatividad del género. En este sentido, el objetivo establecido para el estudio consistió en analizar las formas de violencia que afectan a las y los estudiantes que contradicen los estereotipos de la feminidad y la masculinidad hegemónica en dos grados de décimo del colegio Paulo Freire.

Para llegar a tal punto, la investigación se basó en la metodología cualitativa. Teniendo en cuenta lo postulado por Roberto Hernández (2014), a través de esta metodología se puede reconstruir la realidad mediante los puntos de vista de las personas que hacen parte de un contexto específico. Por ello tiene, por un lado, un carácter interpretativo que se orienta a las prácticas y pensamientos de las personas con la finalidad de entender sus significados, de tal manera que permita otorgarle sentido a los fenómenos que acontecen. Por otro lado, tiene un carácter naturalista, en la medida que analiza los fenómenos y las personas en los ambientes donde transcurren.

Así pues, el trabajo desarrollado se llevó a cabo en el Colegio Distrital Paulo Freire, ubicado en la localidad de Usme, Bogotá, una zona que se caracteriza por albergar parte de los sectores populares de la capital colombiana. En este sentido, la comunidad estudiantil que asiste a dicha institución pertenece a los estratos socioeconómicos uno, dos y tres. La población que fue partícipe como objeto de estudio comprendió a ochenta estudiantes adolescentes, cuyas edades rondaban entre los dieciséis y dieciocho años. La mitad pertenece al género femenino y la otra mitad al masculino, ambos géneros con la orientación sexual heterosexual. Los instrumentos aplicados a dicha población fueron, en primera instancia, el cuestionario. Este se entiende como una técnica de 
investigación cuantitativa que consiste en el planteamiento estructurado de una serie de interrogantes para obtener una determinada información sobre la muestra (Meneses y Rodríguez-Gómez, 2011). El cuestionario surgió tras la realización de una serie de talleres didácticos que abarcaban cuestiones concernientes a la temática del género. Se utilizó para identificar, por un lado, los estereotipos de género que circulan en el estudiantado y, por el otro, los y las estudiantes que existen desde masculinidades y feminidades no estereotipadas. Una vez identificada la población de interés, se aplicó sobre ella el segundo instrumento, la entrevista. Esta representa una de las técnicas más utilizadas en la metodología cualitativa y, en términos generales, consiste en el diálogo entablado por dos o más personas para profundizar acerca de las percepciones del objeto de estudio (Meneses y Rodríguez-Gómez, 2011). Tal instrumento fue aplicado a cuatro estudiantes, dos varones y dos mujeres, quienes representaron otras formas de ser mujer y ser hombre. Mediante la conversación se logró conocer las violencias que enfrentan en el escenario escolar para construir su identidad de género de una forma diferente a los parámetros convencionales.

En lo que respecta al proceso analítico, se hizo uso del software Atlas.ti. Este es un programa informático para el análisis de datos cualitativos que permite trabajar con una variedad de información, como lo son textos, imágenes, audios, videos, etc. (Varguillas, 2006). Con la utilización del Atlas.ti, los datos fueron sistematizados y codificados. Luego se entablaron relaciones entre estos que permitieron, por último, la elaboración de categorías y redes semánticas (esquemas). En este sentido, la construcción del conocimiento fue de carácter inductivo, pues se estableció la teoría a partir de los hallazgos.

\section{¿Cuál es el sistema de dominación que fija las diferencias de sexo, género y sexualidad?}

El desarrollo teórico del feminismo a partir de la tesis planteada por Simone De Beauvoir (2014) "no se nace mujer: se llega a serlo" (p. 207), conllevó a develar el supuesto carácter natural de la jerarquía de los sexos, así como los atributos supuestamente esenciales de la feminidad que daban muestra de la inferioridad de las mujeres con respecto a los varones. Aunque dicho planteamiento fue importante para su tiempo, tal como lo afirma Mara Viveros (2016), no logró describir las experiencias opresivas que atravesaban todas las mujeres, sino más bien un grupo de ellas, aquellas que ostentaban algunos privilegios sociales.

El sesgo analítico de dichas feministas por identificar y comprender las diferencias existentes dentro del presunto grupo cohesionado de mujeres conllevó a enarbolar una lucha teórica y política por la liberación femenina, justificada a partir de una opresión compartida (Lugones, 2006). Este proceso resultó 
contraproducente a lo que aspiraba, en la medida en que silenció, invisibilizó y subordinó las experiencias opresivas particulares de las mujeres de color, las proletarias y las lesbianas, con lo que quedó claro que el proyecto de liberación estaba dirigido por y para las mujeres blancas con privilegio de clase.

Como resultado, se desarrollan otras corrientes feministas que se oponen a los postulados del feminismo hegemónico con el fin de superar la concepción constructivista que aísla el género de las otras clasificaciones sociales y, así, poder hablar del funcionamiento de los sistemas opresivos de una forma entrecruzada que permita el surgimiento del enfoque teórico interseccional. Un ejemplo temprano de dicho pronunciamiento fue realizado por el feminismo negro de los Estados Unidos en los años ochenta, cuyo proyecto político se basó en la "construcción de un movimiento social sensible a todos los tipos de opresión, exclusión y marginación: clasismo, sexismo, racismo, heterosexismo, sin priorizar ninguno de ellos de antemano, sino en forma contextual y situacional" (Viveros, 2016, p. 13).

Entre las corrientes surgidas por la fractura del feminismo hegemónico para abordar la realidad de otras mujeres, se encuentra el lesbofeminismo. La aportación teórica de este consistió en la comprensión de la heterosexualidad, no como una simple orientación sexual, sino, más bien, como un sistema que gobierna la sociedad y permite la subordinación de las mujeres frente a los varones.

En el año de 1980, la poeta Adriaenne Rich (1996) escribe el ensayo Heterosexualidad obligatoria y existencia lesbiana, no con la intención de reproducir las divisiones del movimiento feminista, sino con la finalidad de "animar a las feministas heterosexuales a analizar la heterosexualidad como institución política que debilita a las mujeres, y a cambiarla" (p. 15). Para Adrianne la heterosexualidad es una forma de dominación masculina sobre las mujeres. Por ello, establece el término "heterosexualidad obligatoria" para referirse a los diversos medios por los cuales se busca que la mujer oriente su deseo hacia el varón. En este sentido, la heterosexualidad es una orientación sexual impuesta solo para las mujeres con la finalidad de que los varones puedan tener el control sobre el campo afectivo y sexual de ellas. $Y$ al igual que los sistemas opresivos del racismo o el capitalismo, la heterosexualidad se sostiene a partir de las fuerzas de la violencia física y la falsa conciencia, lo cual ha "(convencido a las mujeres de que el matrimonio y la orientación sexual hacia los hombres son componentes inevitables en sus vidas, por más insatisfactorios u opresivos que resulten" (Rich, 1996, p. 27).

Siguiendo una línea parecida, y desde un enfoque materialista, la teórica francesa Monique Wittig (2006) también concibe la sexualidad heterosexual como una imposición que solo recae en las mujeres. En este sentido, son ellas 
las que son heterosexualizadas, pues así no solo se asegura la reproducción de la especie, sino también del orden heterosexual. La reproducción es la forma de explotación de la economía heterosexual, en la medida en que son los varones los que se apropian del trabajo realizado por las mujeres, lo cual no solo implica reproducir, sino también producir. Esto se traduce en la crianza de las hijas y los hijos en relación con la realización de labores domésticas (en este sentido, la explotación de la economía heterosexual es comparable con la explotación de la economía capitalista, en la medida en que es la clase burguesa la que se apropia de la plusvalía producida por la clase proletaria). Para llevar a cabo dicha empresa, se estableció el contrato social del matrimonio. Por medio de dicha unión la mujer, junto con su reproducción y producción, pasa a ser propiedad de su marido.

Si bien tales afirmaciones son relevantes para un análisis crítico de la heterosexualidad, es necesario avanzar sobre dicha concepción, ya que suponer que la heterosexualidad es una obligación que concierne al género femenino equivale a afirmar que los varones nacen con el deseo heterosexual, es decir, que son heterosexuales per se, lo cual desdibuja por completo la relación del dominio heterosexual con el género masculino. Las conceptualizaciones en torno a la masculinidad nos pueden dar luces sobre la cuestión, pues la construcción de la virilidad implica excluir el deseo homosexual (Colina, 2009; Nascimiento, 2014), de manera que una de las formas en las cuales se forja la masculinidad es mediante el condicionamiento de ser heterosexual.

Continuando con el desarrollo conceptual que ofrece Wittig (2006), su perspectiva frente al sexo como una categoría que traza la división entre los varones y las mujeres, le permite argumentar que los sistemas de dominación se basan en la construcción de diferencias establecidas en el plano económico, político e ideológico. Estas diferencias son representadas como naturales, es decir, precedentes a la cultura y, por ende, condicionantes del orden social existente, con lo que se logra legitimar las desigualdades sociales. Lo anterior lleva a la autora a concluir que "es la opresión la que crea el sexo, y no al revés" (Wittig, 2006, p. 22).

Atendiendo a su planteamiento, se decide indagar en la población estudiantil los estereotipos de género, es decir, las diferencias concebidas entre las mujeres y los varones (figuras 1 y 2 ). 


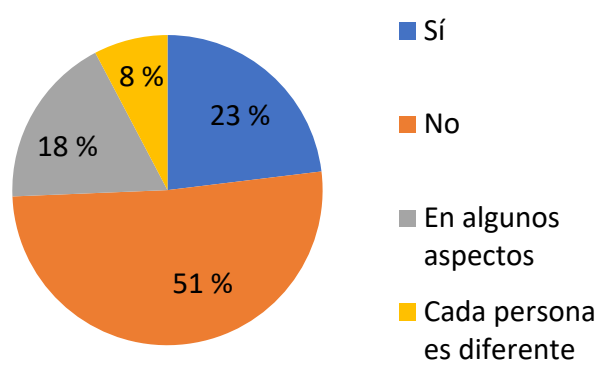

Figura 1. ¿Existen diferencias entre los varones y las mujeres?

Respuesta de la población femenina

Fuente: elaboración propia.

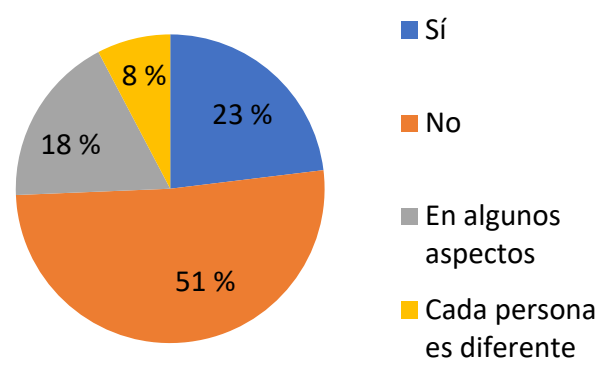

Figura 2. ¿Existen diferencias entre los varones y las mujeres?

Respuesta de la población masculina

Fuente: elaboración propia.

En cuanto a las argumentaciones de las respuestas, se puede mencionar que las diferencias que se establecieron entre las mujeres y los varones fueron principalmente: el físico, el pensamiento y los gustos. Al ser el físico el aspecto más mencionado, puede interpretarse que la diferencia más representativa que tiene el estudiantado en cuanto a las mujeres y los varones no posee relación con el género, sino con el sexo. Este se entiende como las distinciones anatómicas que permiten identificar el físico, tal como lo menciona el estudiantado, es decir, el dimorfismo sexual que puede contemplarse. En lo que compete a las otras dos diferencias, pensamiento y gustos, tienen relación con el género en la medida en que ambos aspectos no son consecuencia del campo biológico, sino más bien del campo sociocultural, pues el género supone un "conjunto de prácticas, creencias, representaciones y prescripciones [...] en función de una simbolización de la diferencia anatómica entre hombres y mujeres" (Lamas, p.3, 2000). Por lo tanto, las distinciones entre pensamientos y gustos hacen parte de una creencia cultural, y al ser parte de la cultura, siguiendo la misma línea de la autora, se torna como un conocimiento tácito y, por ende, como una verdad. 
Por otro lado, aquellos y aquellas estudiantes que dijeron que los hombres y las mujeres no son diferentes, afirmaron que las mujeres y los hombres pueden tener los mismos gustos, hacer las mismas cosas, pensar de la misma manera y que las diferencias son establecidas por la sociedad. Esto resulta bastante interesante, pues se distancia de la idea tradicional de la diferenciación cuando se contempla un escenario posible de puntos de encuentro entre el hombre y la mujer, y de paso desnaturaliza la idea de la diferencia al admitir que esta es consecuencia del factor social. Esta idea se aproxima a la afirmación de que "las variantes comportamentales, sentimentales y de pensamiento se atribuyen más a la influencia de la cultura. Se estima que unos y otras tienen las mismas emociones y sentimientos, y potencialmente la misma capacidad mental" (Aguilar et al., 2013, p. 208). Con relación a la afirmación de que todas las personas son diferentes, se puede concluir que para algunos/as estudiantes la diferencia de las gentes no se basa en el género, sino en el propio carácter individual de cada persona.

Ahora bien, cabe formular la pregunta: ¿Cuál es ese sistema de dominación y pensamiento dominante que crea y justifica las diferencias en relación con el género? Para Wittig (2006) no se trata más que de la heterosexualidad, la cual crea la categoría sexo y, con ello, la dicotomía macho/hembra, masculino/ femenino, lo que permite el establecimiento de las relaciones de poder entre los varones y las mujeres. Más aún, no solo establece la diferencia en cuanto género, sino también por la sexualidad. Así, la lesbiana y el gay también terminan siendo subalternizados/as bajo este sistema, y el abanico de personas oprimidas se expande con las múltiples formas expresivas del género y sexualidad que contradicen los mandatos heteronormativos. Para que dicho sistema se derrumbe, Wittig (2006) introduce la dialéctica en la relación de poder entre el hombre y la mujer al postular que las mujeres deben librar la una lucha para "hacer desaparecer a los hombres como clase, no con un genocidio, sino con una lucha política. Cuando la clase de los "hombres» hayan desaparecido, las mujeres como clase desaparecerán también, porque no habrá esclavos sin amos" (p. 38). Así, la liberación de las y los oprimidos/as por la heterosexualidad llegará cuando la categoría sexo haya sido destruida. Por ello, cree que una persona homosexual que se reivindique como hombre o como mujer legitima la heterosexualidad. En este sentido, apoya, más bien, las identificaciones de lesbiana y de gay porque rechazar la heterosexualidad implica a su vez rechazar el sexo. En pocas pablaras, dice que las y los homosexuales no son ni varones ni mujeres, ya que se es mujer y varón en tanto se es heterosexual. Esta es una idea común en nuestra sociedad, donde se entremezclan las categorías de género y sexualidad. Así, por ejemplo, un estudiante entrevistado afirmó lo siguiente tras haberle preguntado si le habían dicho cómo ser hombre: 
No, pues de pronto mis papás dicen que en tener relaciones sexuales, que así es como se mide un hombre, teniendo relaciones sexuales. Digamos mi papá dice que a los 16, a la edad que yo tenía no era virgen, y yo todavía soy virgen, entonces dice que porque tampoco yo me he desarrollado, que soy muy prematuro y eso, entonces dice que tengo una mentalidad de muy niño, entonces piensan que me voy a volver muy niña, por decirlo así a veces. (Estudiante masculino, comunicación personal, 1 de octubre del 2018)

Atendiendo al último planteamiento de la autora, se ha generado un cuestionamiento presente en el ejercicio investigativo ¿Afirmarse como homosexual no sería también legitimar otra diferencia establecida por la misma heterosexualidad? Podría pensarse que Wittig (2006) cae en una trampa. Aunque lucha por acabar con la categoría sexo, ya que es la idea naturalizada de la diferencia que fundamenta la dominación heterosexual, se declara a favor de la categoría de sexualidad, por ello no se reconoce como mujer, pero sí como lesbiana. Sin embargo, el hecho de afirmarse como homosexual reivindica otra diferencia que también es producto de la misma hegemonía heterosexual y con la cual también funciona. Como lo recuerda Sedgwick (1998), antes de que apareciera el concepto heterosexual, primero surgió el de homosexual. Por tanto, la heterosexualidad se fundamenta a partir de su diferencia, de aquello que no es o que no debería ser.

Entre las pensadoras que fueron influenciadas por la anterior autora, se destaca la filósofa Judith Butler (2007), quien elabora una de las obras más significativas para la teoría feminista y queer, El género en disputa, siguiendo otras intelectuales de Francia-. Allí describe la categoría de la matriz heterosexual como el campo en el que se producen identidades inteligibles, es decir, aquellas que mantienen la coherencia entre el sexo, el género y la sexualidad. Esto supondría, entonces, que los géneros culturales masculino y femenino son el reflejo de los sexos biológicos de macho y hembra, ambos concebidos como categorías opositoras y complementarias por el deseo heterosexual; opositoras por la diferencia que poseen entre sí y complementarias por la unión en la práctica sexual. De esta manera, el deseo expresa el género o, si se quiere, el género expresa el deseo. Por tanto, la exclamación "me gustan las mujeres", daría entender que el enunciado es pronunciado por un sujeto masculino, mientras que la afirmación "soy mujer" daría entender un deseo dirigido hacia los varones. En definitiva, "instituir una heterosexualidad obligatoria y naturalizada requiere y reglamenta al género como una relación binaria en la que el término masculino se distingue del femenino, y esta diferenciación se consigue mediante las prácticas del deseo heterosexual" (Butler, 2007, p. 81).

Dicho postulado puede ejemplificarse con la presentación de las siguientes redes semánticas que fueron elaboradas con ayuda del programa de Atlas. ti, las cuales nos presentan de una manera breve y esquemática los datos (figuras 3, 4 y 5). 


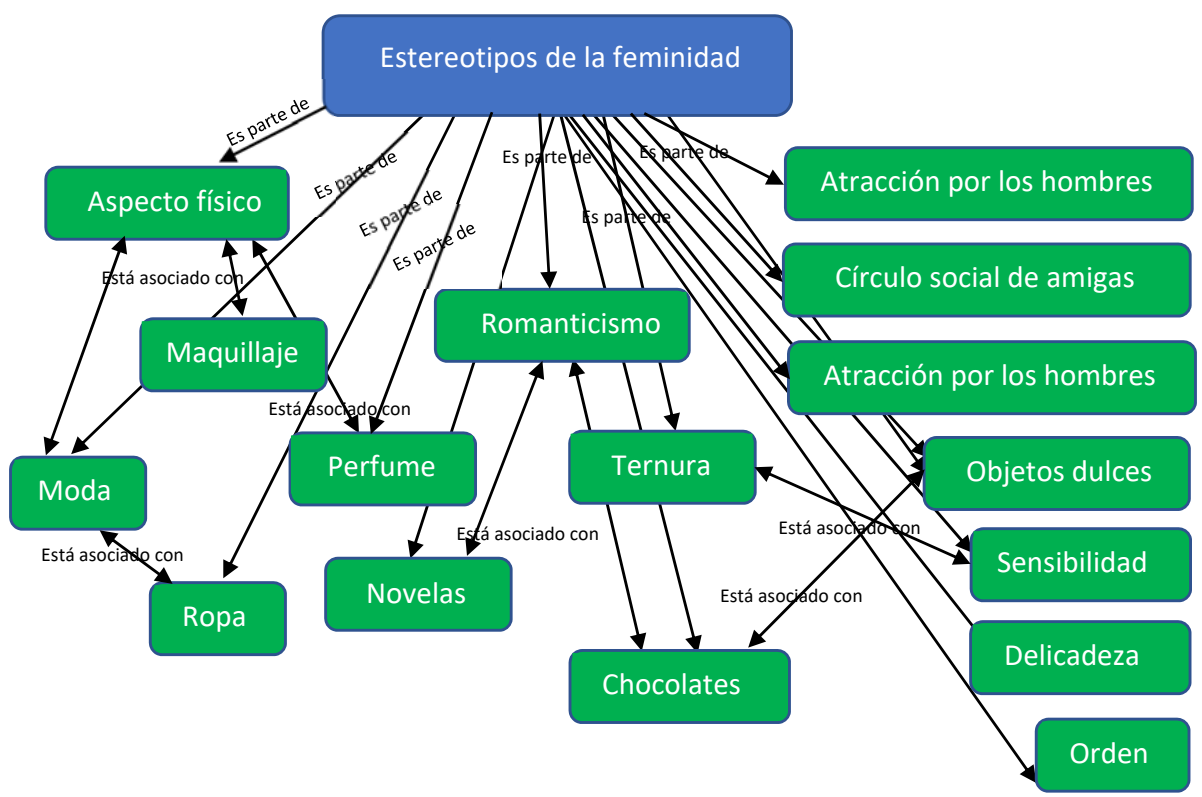

Figura 3. Estereotipos de la feminidad

Fuente: elaboración propia.

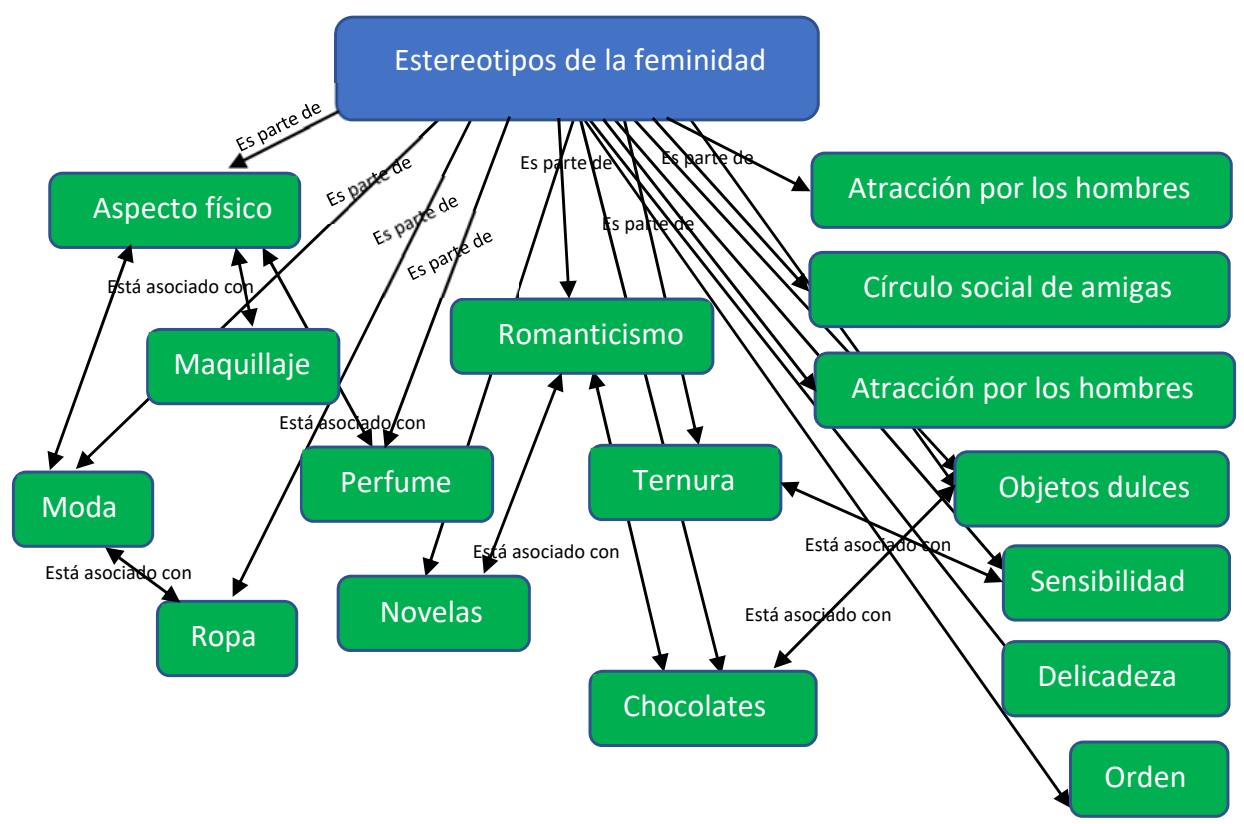

Figura 4. Estereotipos de la masculinidad

Fuente: elaboración propia. 


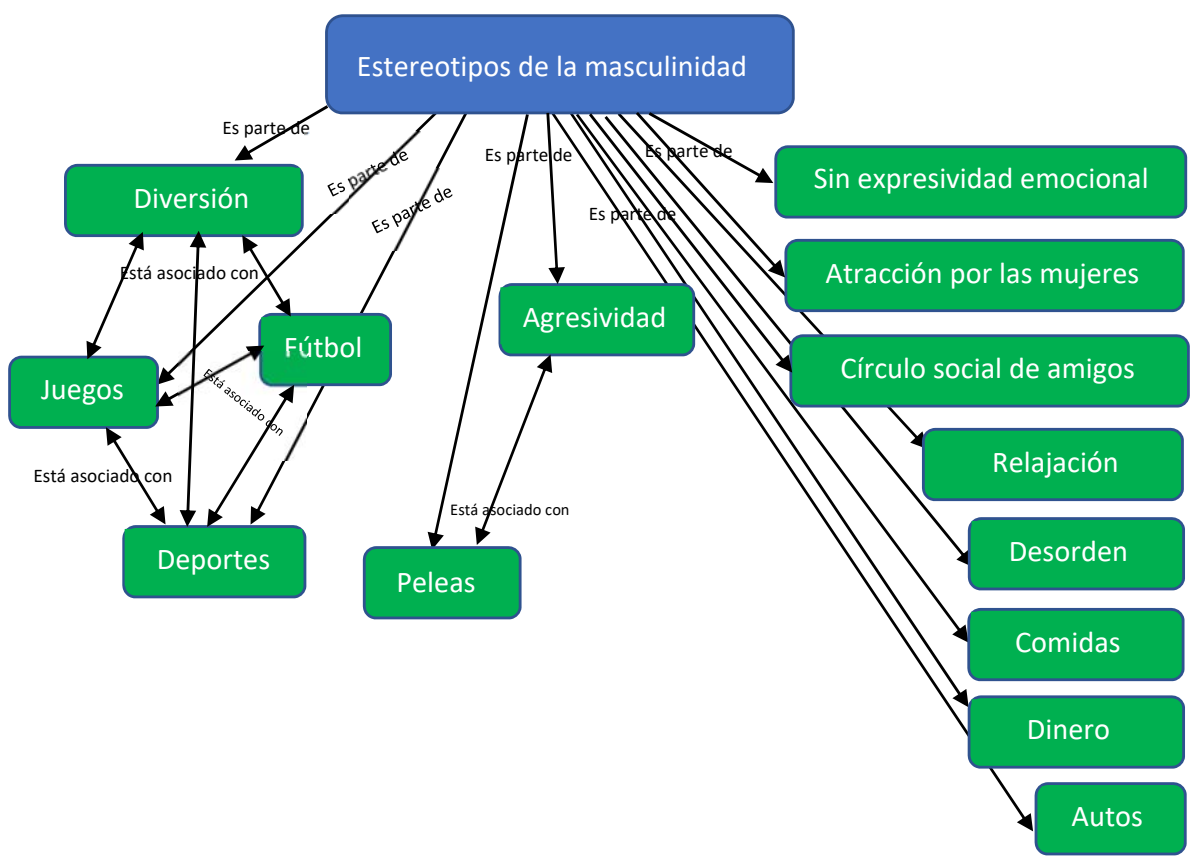

Figura 5. Estereotipos de la heterosexualidad

Fuente: elaboración propia.

Tal como se puede observar con estos tres esquemas, se asignan cualidades que resultan completamente contradictorias entre los varones y las mujeres. Sin embargo, poseen una característica en común, un punto de encuentro entre ambas polaridades: la atracción por el supuesto género opuesto, es decir, su deseo heterosexual.

Tal como lo señala Butler (2007), así como la matriz heterosexual establece la coherencia en la identidad, también define aquellas imposibilidades o inviabilidades de la existencia. De esta manera, los individuos que no se adaptan a la normativa heterosexual que regula el género y la sexualidad (homosexuales, intersexuales, transexuales, etc.) quedan etiquetados bajo el epíteto de abyección, y, por ende, reducidos en términos sociales. Entre el mosaico de las personalidades abyectas, podríamos señalar a las mujeres masculinas y a los varones femeninos, quienes ponen en contradicción, mediante sus actos, las ideas frente al deber ser de un hombre y de una mujer.

En este sentido, las feminidades y masculinidades que no responden a los estereotipos tradicionales también serían víctimas de formas específicas de violencia en lo que respecta al género, de allí que fuese interés de estudio. Para ello, se tomó el aporte conceptual que realiza Johan Galtung (1990) respecto 
a la clasificación de las violencias. Para este sociólogo existen tres formas de violencia: la estructural, la directa y la cultural (Galtung, 1990). En la primera forma se lleva a cabo una explotación, es decir, una situación donde se privilegia los intereses de un determinado grupo social por encima de otro, lo que genera relaciones desiguales en la medida en que un grupo tiene mejores condiciones de existencia que otro. Siguiendo al presente autor, Leyton y Toledo (2012) señalan que el tipo de violencia directa se refiere a aquellas acciones que permiten la destrucción de un ser vivo, y se caracteriza por materializarse de forma inmediata y por evidenciarse fácilmente. La mayor expresión de esta violencia es la guerra, ya que se puede apreciar fácilmente el exterminio de la existencia humana. En lo que respecta a la violencia cultural, como lo describe Galtung (1990), se trata de un medio simbólico para la justificación de las dos anteriores expresiones de violencia. En este sentido, permite su naturalización y aceptación por parte de la sociedad. La violencia cultural se conforma por la religión, la ideología, el lenguaje, el arte, la ciencia empírica y formal y, por último, la cosmología.

Así pues, el primer tipo de violencia se ejerce desde el Estado y generan las desigualdades sociales. El segundo se realiza sin ninguna intervención entre la persona que violenta y la que es violentada. Este tipo de violencia se conforma, a la vez, por dos formas de violencia: la verbal, donde se afecta a alguien mediante el lenguaje, y la física, donde se agrede a alguien por la fuerza física o por la utilización de un instrumento (como por ejemplo un arma). Por último, el tercer tipo de violencia se caracteriza por legitimar las dos formas de violencia antes señaladas en el plano simbólico, lo que permite la naturalización de acciones de corte violento (Galtung, 1990). Esta legitimación puede efectuarse por el conjunto de "las ideas, las normas, los valores, la cultura, la tradición" (Jiménez, 2010, p. 37) que se reproduce en una sociedad sin cuestionamiento alguno.

Ahora bien, teniendo presente la anterior categorización, se hace menester identificar aquellas formas violentas que se manifiestan en el escenario escolar con relación a la homofobia, pues esta categoría, tal como lo menciona Salvador Cruz (2002) y Carlos Colina (2009), no solo consiste en la violencia que se ejerce por parte del sector heterosexual hacia el homosexual (o a cualquier individuo abyecto utilizando el término acuñado por Butler), sino también a aquellos sujetos que no recrean los estereotipos hegemónicos de la feminidad y la masculinidad. En dicho sentido, la homofobia no solo es un ejercicio de violencia dirigido hacia las personas que poseen la inclinación sexual hacia el mismo sexo o hacia las que desestabilizan el binarismo de sexo/género, tal como lo son las personas intersexuales o trans. Por el contrario, la violencia también funciona como un medio para coaccionar las prácticas de las personas en general. Por lo tanto, las personas heterosexuales que no cumplen con los mandatos normativos del 
género también se ven envueltas por esta dinámica violenta, por lo que puede identificarse la homofobia como el mecanismo propio de la heteronormatividad.

Por las entrevistas realizadas, se halló que se presencia la forma de violencia directa y la violencia cultural, las cuales se pueden englobar en otras dos formas de violencia, a saber, la homofobia y el sexismo.

En lo que respecta a violencia directa de corte homofóbico, se evidencia que el estudiantado que presenta otra forma de ser hombre o mujer es agredido/a verbalmente por parte de sus compañeras y compañeros de clase, así como por estudiantes de otros cursos. Tales estudiantes han sido señaladas y señalados con peyorativos como "marimacha", "marica" y "machorra". Asimismo, también se les ha llamado "hombre" a las mujeres, y "niña", "mujer" y "gay" a los hombres, representando tales identidades como ofensas. Así lo argumentaron tres de cuatro estudiantes entrevistados/as tras realizarles la pregunta: ¿Has escuchado los términos "marica" o "marimacha" en el colegio?

Sí, más que todo acá en el colegio, porque digamos hay muchos hombres que, o sea digamos, cada uno tiene su manera de ser y por ejemplo hay muchos hombres que si están mal demuestran estar mal, algunos, entonces como que si lo ven mal "ay no sea marica" o digamos muchos hombres que se la pasan digamos también con mujeres porque prefieren las amistades de mujeres, entonces que por eso es marica. Digamos muchas mujeres, como le digo, porque juegan y eso dicen que es marimacha, o también hay muchas mujeres que como tal actúan como hombres a la hora de hablar y eso, no solo respecto a las groserías sino como digamos "ay ñero, que no sé qué", ellos lo consideran como, si actúa como hombre entonces es una marimacha. Son como en esos casos en que muchas veces los hombres demuestran actitudes de mujeres y nosotras actitudes de hombres. (Estudiante femenina, comunicación personal, 28 de septiembre del 2018)

Sí, bastante. Digamos el año pasado había un niño que era gay de once y todo el mundo, "vea el gay que no sé qué" o también cuando hay una mujer que juega fútbol y que tiene las piernas gordas y eso "vea la marimacha que no sé qué", o cuando un hombre es muy atraído a las mujeres y empieza a jugar con ellas, le dicen que es como gay. Yo viví eso como en séptimo, me la pasaba mucho con niñas, también me dijeron eso. (Estudiante masculino, comunicación personal, 1 de octubre del 2018)

No solo se han presentado agresiones verbales, sino también físicas. Así lo aseguró un estudiante entrevistado cuando dijo que

a mí digamos me pegan en el salón a veces y pues piensan que a uno no le duele, y medio me hacen así con esto y tienen esto grueso [los nudillos] entonces me duele y ellos dicen que no y muestran con una mujer y le pegan y dicen que no, que no les duele, pues obvio porque le van a pegar más pasito. (Estudiante masculino, comunicación personal, 1 de octubre del 2018) 
De esta manera, se observa que la violencia directa se manifiesta tanto verbal como físicamente por la lógica de violencia homofóbica. Aunque se pensaría que su materialización radica en una cuestión de orientación sexual, los hallazgos demuestran que el fundamento de la homofobia, en este caso, se relaciona con una cuestión de género.

Ahora bien, debe mencionarse que dichos estereotipos de género son ideas sexistas que se encargan de promover la distinción entre las personas debido al sexo. Dichos estereotipos no se quedan en el plano abstracto de las ideas, ya que, como lo recuerdan Felitti y Rizzotti (2016), dichas representaciones poseen la fuerza de influenciar las conductas humanas, es decir, de tener efectos materiales. De allí la importancia de haber presentado las redes semánticas, pues no solo refleja estereotipos del género femenino y masculino, sino también la forma en la que la mujer y el varón deben accionar. Estos estereotipos reflejan el sexismo que permanece en el plano mental de las personas. Por lo tanto, y siguiendo a Galtung (1990), el sexismo se configura como una violencia simbólica al naturalizar las representaciones sociales del género que, al ser puestas en tela de juicio por prácticas que desafían los mandatos heteronormativos, conlleva al despliegue del ejercicio de violencia para instaurar ciertas prácticas genéricas en determinados cuerpos sexuados. Por lo tanto, el sexismo funciona como base ideológica para justificar la materialización de la homofobia. Para realizar un resumen de los datos hallados con respecto a la identificación y clasificación de violencias del sistema heteronormativo en dicha cultura escolar, se elaboró el siguiente esquema (tabla 1):

Tabla 1. Manifestaciones de violencia propias de la heteronormatividad

\begin{tabular}{lllc}
\cline { 2 - 3 } & \multicolumn{2}{c}{ Heteronormatividad } & Sexismo \\
\cline { 2 - 4 } & \multicolumn{2}{c}{ Homofobia } & Violencia cultural \\
\cline { 2 - 4 } & \multicolumn{2}{c}{ Violencia directa } \\
\hline Género & Verbal & Física & Estereotipos femeninos \\
\hline Femenino & Marimacha, machorra, hombre. & Golpes & Estereotipos masculinos \\
\hline Masculino & Marica, niña, gay, mujer. & C.
\end{tabular}

Fuente: elaboración propia.

\section{Conclusiones}

Mediante el ejercicio investigativo realizado, se logró relacionar el planteamiento de Louis Althusser (2003) con la escuela que, más que un mero aparato que permite la reproducción del sistema de dominación capitalista, se concibe como una institución social en la que se reproducen relaciones de poder que 
están más allá de la clase (pero que mantienen relación con ella) y, al mismo tiempo, como un espacio de resistencia frente a dichas formas de dominación. Para el presente estudio, nos enfocamos en identificar las formas de violencia que produce la heteronormatividad para evidenciar una de las formas en las que se reproduce dicho sistema en una escuela particular de Bogotá. Mediante el uso de la encuesta y la entrevista como las herramientas para recolección de datos, se halló que el estudiantado que hace parte de formas masculinas y femeninas que no responden a los estereotipos tradicionales, se ven violentados/ as por parte de sus compañeros/as por no materializar varios de los estereotipos de su género o por materializar aquellos que no le son "propios" de su género. Teniendo presente la clasificación que realiza Johan Galtung (2003) respecto a las formas de violencia, se evidenció que se presenta la violencia directa, la cual se hace visible por medio de insultos y golpes, así como la violencia cultural, que se presencia mediante los estereotipos de género. Cabe resaltar que estas dos formas de violencia se efectúan o se circunscriben bajo otros dos tipos de violencia más generales. Así pues, la violencia directa es producto de la homofobia, puesto que esta no solo se ejerce hacia las personas que poseen gusto por el mismo sexo o aquellas que quebrantan el binarismo sexo/género, sino también, como lo señalan Carlos Colina (2009) y Salvador Cruz (2002), hacia las personas heterosexuales que incumplen la normativa del género. En cuanto a la violencia cultural, se evidencia que su aparición se debe al sexismo, pues el mantenimiento de representaciones mentales que distinguen al varón de la mujer justifica prácticas homofóbicas hacia aquellos sujetos que no materializan las ideas concernientes a su propio género mediante sus actos.

De esta manera, se demuestra que en la escuela se legítima el sistema heteronormativo al materializarse formas de violencia que promueven estándares rígidos y limitantes en lo que respecta a la forma de ser un varón o ser una mujer. Esta problemática, así como otras que generalmente pasan inadvertidas, deben ser parte de la reflexión pedagógica. Por ello, cabría seguir a Deborah Britzman (2002) para preguntarnos: "¿Es posible que la pedagogía vaya más allá de la producción de posiciones subjetivas esencialistas y que reflexione sobre la formación del sujeto prestando atención a la performatividad de éste desde la relacionabilidad queer?" (p. 197). De tal forma, la pedagogía jugaría un papel clave en la prevención de violencias patriarcales (homofobia y sexismo) manifiestas en la escuela, puesto que serviría como una herramienta para cuestionar las identidades normativas y reflexionar en torno a las prácticas que las refuerzan y las mantienen. Es posible que de dicha forma se logre desnaturalizar los estereotipos de género que circulan en las mentes de estudiantes y docentes y, con ello, solventar los efectos negativos que acarrean para los sujetos que no se encuentran en la categoría de normalidad genérica y sexual. 
Por último, debe mencionarse que se evidencia la necesidad de empezar a trabajar las temáticas de género y sexualidad. Aunque estas no se encuentren presentes en el currículo, se evidencia el interés y la motivación del estudiantado por conocer acerca de dichos ejes temáticos, en la medida en que encuentran un lugar para entenderse a sí mismos, así como a las y los demás. Esto quiere decir que esta temática se presenta un conocimiento contextualizado que desemboca en un aprendizaje significativo.

\section{Referencias}

Althusser, L. (2003). Ideología y aparatos ideológicos del Estado. Freud y Lacan. Nueva Visión.

Aguilar, Y., Valdez, J., González Arraita, N. y Gonzáles, S. (2013). Los roles de

Género de los hombres y las mujeres en el México contemporáneo. Enseñanza e investigación en Psicología 18(2), 207-224. https://www.redalyc.org/pdf/292/29228336001.pdf

Butler, J. (2007). El género en disputa. Edición Paidós Ibérica

Britzman, D. (2002). La pedagogía transgresora y sus extrañas técnicas. En R. Mérida (coord.), Sexualidades transgresoras: una antología de estudios queer (pp. 197-228). Icararia.

Cantor, E. (2009). Cultura estudiantil y diversidad sexual discriminación y reconocimiento de los y las jóvenes LGBT en la secundaria. Polisemia, 5(8), 101-110. https://doi.org/10.26620/uniminuto. polisemia.5.8.2009.101-110

Colina, C. (2009). La homofobia: heterosexismo, masculinidad hegemónica y eclosión de la diversidad sexual. Razón y palabra, (67), 1-23. https://www.redalyc.org/articulo.oa?id=199520725011

Cruz, S. (2002). Homofobia y masculinidad. El Cotidiano, 18(113), 8-14. https://www.redalyc.org/ pdf/325/32511302.pdf

De Beauvoir, S. (2014). The second sex. Penguin Random House.

Felitti, K. y Rizzotti, A. (2016). El "machismo latinoamericano" y sus derivas en la educación internacional: reflexiones de estudiantes estadounidenses en Buenos Aires. Magis, 9(18), 13-28. https:// doi.org/10.11144/Javeriana.m9-18.mlde

Giroux, H. (1983). Teorías de la reproducción y la resistencia en la nueva sociología de la educación: un análisis crítico. Revista Colombiana de Educación (17) https://doi.org/10.17227/01203916.5140

Galtung, J. (2003). Violencia cultural. Gernika Gogoratuz. https://www.gernikagogoratuz.org/wpcontent/uploads/2019/03/doc-14-violencia-cultural.pdf

Galtung, J. (1990). Cultural Violence. Journal of peace research, 27(3), 291-305.

Hirsch, D. y Rio, V. (2015). Teorías de la reproducción y teorías de la resistencia: una revisión del debate pedagógico desde la perspectiva materialista. Foro de educación, 13(18), 69-91. https://dialnet. unirioja.es/servlet/articulo?codigo $=5153340$

Hernández, R. (2014). Metodología de la investigación. McGraw-Hill/Interamericana Editores S.A. de C.V.

Jiménez, B. (2010). Conocer para comprender la violencia: origen, causas y realidad. CONVERGENCIA. Revista ciencias sociales, 19(58), 13-52 https://www.researchgate.net/publication/262469746 Conocer_para_comprender_la_violencia_origen_causas_y_realidad 
Lamas, M. (2000). Diferencia de sexo, género y diferencia sexual. Cuicuilco. Revista de Ciencias Antropológicas, 7(18), 95-118. https://revistas.inah.gob.mx/index.php/cuicuilco/article/view/360

Leyton, I. y Toledo, F. (2012). A propósito de la violencia: reflexiones acerca del concepto [tesis de pregrado, Universidad de Chile]. Repositorio Académico de la Universidad de Chile. http://repositorio. uchile.cl/handle/2250/116376

Lugones, M, (2003). Multiculturalismo radical y feminismo de mujeres de color. Revista Internacional de Filosofía Política, (25), 61-75. https://www.redalyc.org/pdf/592/59202503.pdf

Meneses, J. y Rodríguez-Gómez, D. (2011). El cuestionario y la entrevista. Universitat Oberta de Catalunya. http://femrecerca.cat/meneses/publication/cuestionario-entrevista

Nascimiento, M. (2014). Hombres, masculinidades y homofobia: apuntes para la reflexión desde lo conceptual y de lo político. Conexões PSI, 2(2), 41-59. https://core.ac.uk/download/pdf/229105373.pdf

Rich, A. (1996). Heterosexualidad obligatoria y existencia lesbiana. Duoda, 10, 15-41. https://www. raco.cat/index.php/DUODA/article/view/62008

Sedgwick, E. (1998). Epistemología del armario. Ediciones de La Tempestad.

Varguillas, C. (2006). El uso de Atlas. Ti y la creatividad del investigador en el análisis cualitativo de contenido UPEL. Instituto Pedagógico Rural el Mácaro. Laurus, 12, 73-87. https://www.redalyc. org/pdf/761/76109905.pdf

Viveros, M. (2016). La interseccionalidad: una aproximación situada a la dominación. Debate feminista, 52, 1-17. http://dx.doi.org/10.1016/j.df.2016.09.005

Wittig, M. (2006). El pensamiento heterosexual y otros ensayos. Cervantes. 